\title{
Computational Study of Graphene- and Few Layer Graphene-Metal Composites Induced by Radiation
}

\author{
Arkady M. Ilyin, Renata R. Nemkaeva and Vladislav V. Kudrjashov \\ National Nanolaboratory, Kazakh National University, Almaty, Kazakhstan
}

Received: January 09, 2013 / Accepted: February 06, 2013 / Published: April 25, 2013.

\begin{abstract}
Nanomaterials, based on few-layer graphene are considered as promising ones in different technological fields relating to materials science, hydrogen energetic and electrical sources, in particular, lithium -ion rechargeable batteries. The paper presents results of computational study of modifying effect caused by structural defects, particularly by "bridge-like" radiation defects in few-layer graphene nanostructures. Computer modeling and quantum mechanical calculations predict that few layer graphene-metal $(\mathrm{Cu}, \mathrm{Al}, \mathrm{Li})$ fast bonds can be induced due to production of radiation structural defects.
\end{abstract}

Key words: Few-layer graphene, bridge-like radiation defect, computer simulation, graphene-metal composites.

\section{Introduction}

It is known that graphene and few layer graphene nanostructures are considered as promising materials in production of electronic devices, electrical sources, in particular, lithium-ion batteries, elements of reinforcement in production of composite materials [1-8]. It should be noticed that many difficulties concerning graphene's usage originate from its rather closed $\mathrm{sp}^{2}$ electronic structure. In practice it results in week interfacial bonding between graphene fragments and matrices when using it as filler substance, for example, in decreasing the part of graphene's surface transferring stress between reinforcement particle and matrix under of the composite. This obviously limits the level of ultimate strength that can effectively be transferred from graphene's element to the matrix. It is one of the barriers on the way of wide application of graphene based elements in new materials production. For example, composite material on the base of copper with graphene fragments as filler would be a material which possesses very high heat conductivity combined with high stiffness and strength. On the

Corresponding author: Arkady M. Ilyin, doctor of science, professor, research fields: nanomaterials and electron spectroscopy. E-mail: ilyinar@mail.ru. other hand, $\mathrm{Al}$ based composite with graphene filler would be very useful material because of unique combination of high strength with light weight. Our recent theoretical investigations [4-6] allow to propose that radiation defects in carbon nanostructures like carbon nanotubes and graphene based materials can essentially improve binding ability of graphene's surface with metallic materials due to production of additional fast covalent bonds. Moreover, it has been stated that so called "bridge-like" radiation defects can link together nanostructures of fillers (particularly, few layer graphene fragments) making them and composite itself much more stiffer and stronger and improving isotropy of physical properties, like electroand heat conductivity. Unfortunately, it is not easy to observe radiation defects directly and to make reasonable interpretations of experimental measurements of material's characteristics. In this situation, computer simulation of radiation defects in carbon nanostructures becomes of great importance. Recently in Refs. [1-4] physical and structural characteristics of single vacancies and complex defects, involving only carbon atoms were theoretically studied. It was established that vacancy zone in graphene raveled out in symmetrical way with 
increasing the hexagons parameter from $2.46 \AA$ to $2.72 \AA$. The energy of relaxation of a vacancy's zone was calculated as large as $1.6 \mathrm{eV}$ [4]. Recently nanomaterials based on ultra-thin graphite have also found a use in the technological field relating to production of lithium-ion rechargeable batteries, as possible storing elements for lithium atoms. But there are some obstacles on this way of application, linking for example, with deformation of cells which results in limitation of a charge capacity and the life-time of devices [7, 8]. Taking all above into account, searching the possible ways to improve the relevant properties of few layer graphene nanostructures are of great interest. Obviously, performing the direct experiments with structures mentioned above is rather difficult problem. In this situation, computer simulation becomes a scientific instrument of a large importance. In our paper the energetic and structural characteristics of complex defects in graphene and few-layer graphene involving metallic atoms and clusters have been studied by quantum- mechanical calculations, based on density functional theory (DFT).

\section{Modeling and Calculations}

\subsection{Bridge-Like Radiation Defects}

Recently, we reported results of computer simulations and studies of energetic and structural characteristics of stable radiation defects like vacancies and carbon-carbon dumbbells in graphene and relative nanostructures [4-6]. In this paper, we focus on complex radiation defects in carbon nanostructures, which involve atoms of metals. All calculations were performed by density functional theory with using a procedure of optimization on energy. The first model which we consider is a special configuration, which is called a "bridge-like defect" and consists of two vacancies, faced each other, produced in both of graphene sheets and interstitial carbon atom (i) caught between them (Fig. 1).

The essential feature of this defect is two graphene

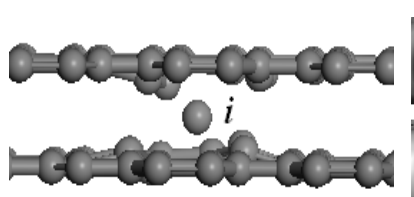

(a)

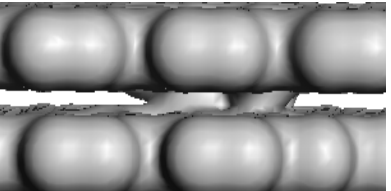

(b)
Fig. 1 A typical configuration of a bridge-like radiation defect. (a) the atomic structure of defect with interstitial $I$, (b) the distribution of the electron charge with the density equal to $1.3 \mathrm{el} / \mathrm{A}^{3}$.

sheets are linked with fast covalent bond. Moreover, neighboring atoms pulling in the gap between sheets, facilitating additional bonds in the zone of the defect. The electron charge distribution calculated for high value of electron density presented in Fig. 1b proves existing of additional bonds between graphene sheets. The total binding energy for this defect was calculated as large as $9.5 \mathrm{eV}$.

\subsection{Graphene-Copper Composition}

Preliminary DFT calculations were performed for metallic atoms $(\mathrm{Cu}, \mathrm{Al}, \mathrm{Li})$ placed in special positions of high symmetry (Fig. 2) on the surface of ideal graphene gave values of the binding energies of metal atoms on the graphene surface nearly zero.

Fig. 3 illustrates a computer model of possible composition $\mathrm{Cu}$, vacancy. In this case calculation gave the binding energy of a $\mathrm{Cu}$ atom as large as $1.1 \mathrm{eV}$ with distance from graphene sheet as large as $1.5 \AA$. Dimension parameters of the vacancy (distances $D_{i j}$ between carbon atoms $1,2,3$ in the graphene sheet) are as follows: $D_{12}=2.91 \AA ; D_{23}=D_{31}=2.96 \AA$.

More complex configuration, a dumbbell of $\mathrm{Cu}$ atoms linked with a vacancy one can see in Fig. 4. Parameters of the vacancy after energy optimization: all three distances are equal: $D_{12}=D_{23}=D_{31}=2.9 \AA$. The $\mathrm{Cu}$ atom binding energy is equal to $1.8 \mathrm{eV}$ with the distance between copper atoms (the length of the dumbbell) equals $2.8 \AA$.

Obviously, production of such defects with metallic dumbbells allows to get higher amount of $\mathrm{Cu}$ in the composition. It should be also noticed that graphene 


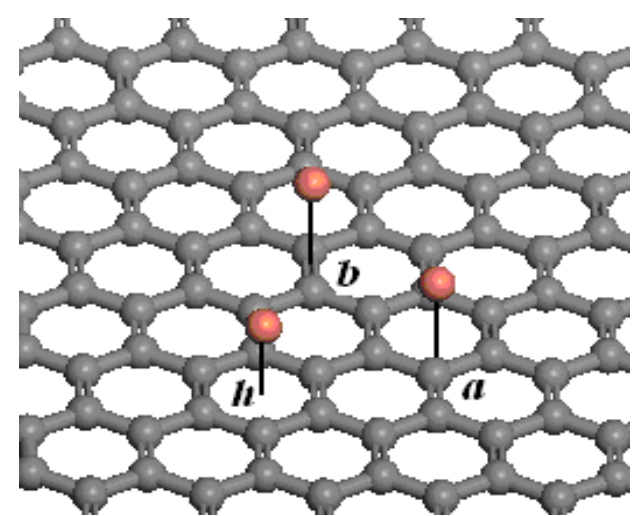

Fig. 2 Possible positions of metallic atoms $(\mathrm{Cu}, \mathrm{Al}, \mathrm{Li})$ arranged on graphene surface in sites of the high symmetry: $h$, over the center of a hexagon; a- over a carbon atom ; b over the center of the $\mathrm{C}-\mathrm{C}$ bond.

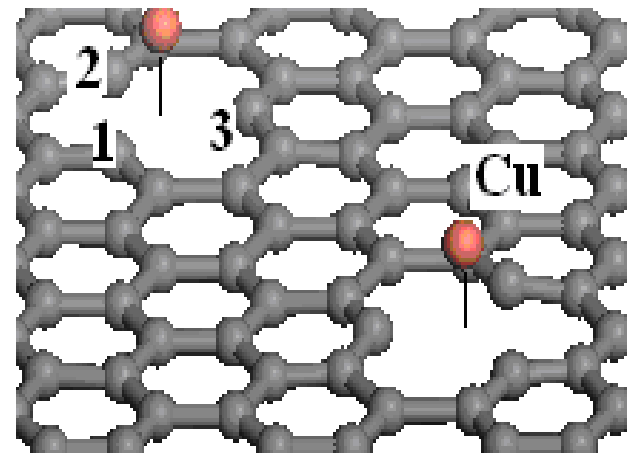

Fig. 3 Single $\mathrm{Cu}$ atoms linked with vacancies in graphene.

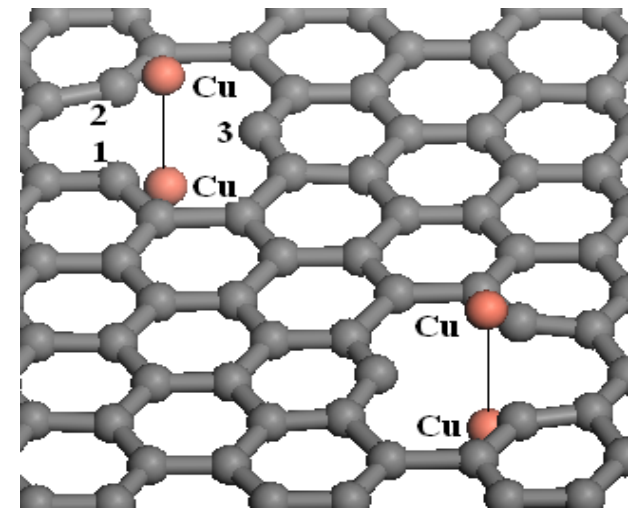

Fig. 4 Graphene with two Cu-dumbbells bonded with vacancies.

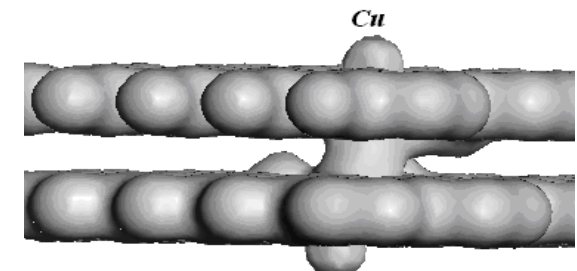

Fig. 5 Bridge-like defect with copper atom attached over vacancies (the distribution of electron charge calculated at density of charge as large as $1.2 \mathrm{el} / \AA^{3}$ ). structure displayed no signs of non-uniformity between the two dumbbells.

Much more complex defect configuration is presented in Fig. 5. It is a bridge like defect, linking two graphene sheets with two attached $\mathrm{Cu}$ atoms on the graphene surfaces. The binding energy of a $\mathrm{Cu}$ atom in this configuration is as large as $1.2 \mathrm{eV}$.

\subsection{Graphene-Aluminium Composition}

The computational model of a relatively simple defect configuration Al, $V$ is presented in Fig. 6. After the procedure of optimization on energy had been performed, the vacancy zone has increased in size and $\mathrm{Al}$ atom occupied its equilibrium position in the graphene's plane. Distances between $\mathrm{Al}$ atom and the nearest carbon atoms (marked as 1, 2, 3) are the same and equal to $1.71 \AA$. The binding energy of $\mathrm{Al}$ with graphene is equal to $3.6 \mathrm{eV}$.

Fig. 7 presents configuration of $\mathrm{Al}$, dumbbell placed at the vacancy. The equilibrium distance between $\mathrm{Al}$ atoms equals $2.5 \AA$, the binding energy is as large as $2.9 \mathrm{eV}$. The electron charge distribution with high electron density proves existing fast bonds between $\mathrm{Al}$ and vacancy.

Fig. 8 shows the bridge-like defect with aluminum atoms attached at the surfaces in the defect area. The binding energy of $\mathrm{Al}$ in this configuration is as large as $1.5 \mathrm{eV}$. The binding energy between grapheme sheets due the single bridge-like defect is as large as $9.3 \mathrm{eV}$.

\subsection{Graphene-Lithium System}

It is well known that ultra-thin graphite and few layer graphene particles can be used as cells for a storage of $\mathrm{Li}$ atoms in lithium ion batteries production technologies. Moreover, the existence of bridge-like defects essentially improves one more important feature of lithium ion sources: it obviously enlarges the permeation and mobility of $\mathrm{Li}$ ions through graphene cells. Therefore, radiation modification of grapheme cells with bridge-like defects might become 


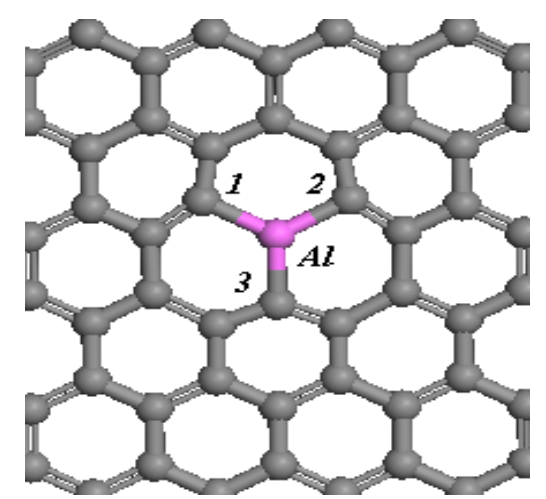

Fig. 6 A 2D defect in graphene involving a vacancy linked with a single $\mathrm{Al}$ atom.

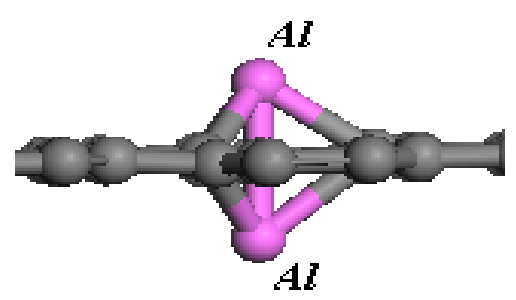

(a)

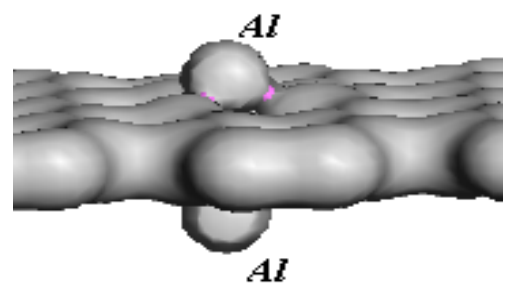

(b)

Fig. 7 A complex dumbbell-like defect of Al atoms in graphene vacancy: (a) atomic structure; (b) the electron charge distribution by the density equal to $1.4 \mathrm{el} / \AA^{3}$.

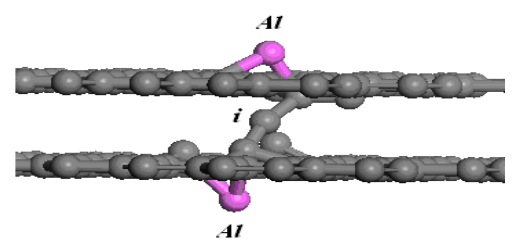

(a)

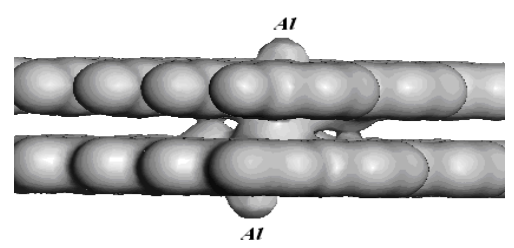

(b)

Fig. 8 A complex "bridge-like defect"-Al atoms configuration in two-layer graphene. (a) The atomic structure, (b) the electron charge distribution.

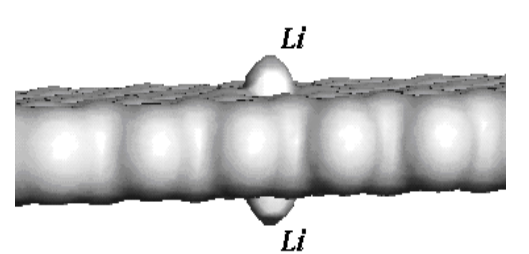

Fig. 9 The electron charge distribution in a Li-dumbbell in graphene's vacancy.

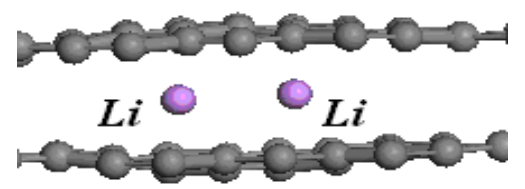

Fig. 10 A computer model of storing lithium atoms between graphene sheets.

a key technology to improve mechanical and electrical properties of lithium ion electrical sources, that results, in particular, in the essential enlargement of charge capacity, dimension stability and life-time of Li-ion-based electrical elements. Fig. 9 shows a possible configuration of a $\mathrm{Li}$-Li dumbbell bonded with a vacancy with binding energy $0.9 \mathrm{eV} /$ atom Li. The distance between $\mathrm{Li}$ atoms and graphene is equal to $1.1 \AA$. The calculation has been performed by the electron charge density equals $0.2 \mathrm{el} / \AA^{3}$. Obviously, this kind of defects can also be in general useful for holding Li by graphene structures.

Obviously, Li atoms diffuse into and out of the electrodes when the battery is charged or discharged. These cycling motion can cause changes in size of lithium storing cells. Therefore, computer simulation of possible dimension changes of graphene cells was also performed. A typical result of modeling presented in Fig. 10 illustrates very important feature of grapheme, based system for storing lithium that can be called as dimension instability. The noticeable deformation of graphene sheets in perpendicular direction can be seen. Similar effects are also possible to be displayed in ultra-thin graphite particles when using for Li storing in lithium ion batteries, This phenomena can significantly limit a capacity of such batteries and cut their life-time by using ultra-thin 


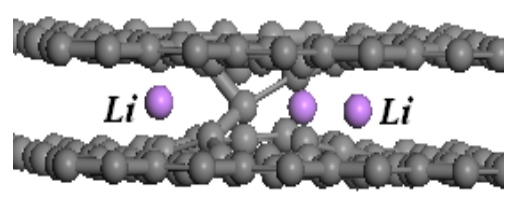

(a)

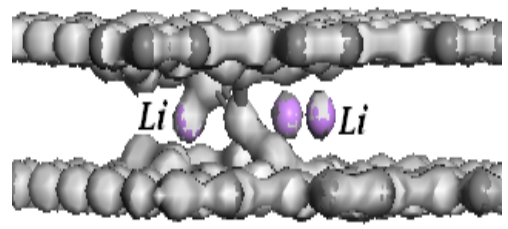

(b)

Fig. 11 Li atoms stored in a two layer graphene cell, with a bridge-like radiation defect. (a) The atomic structure of defect; (b) the electron charge distribution at the charge density equals $0.6 \mathrm{el} / \AA^{3}$.

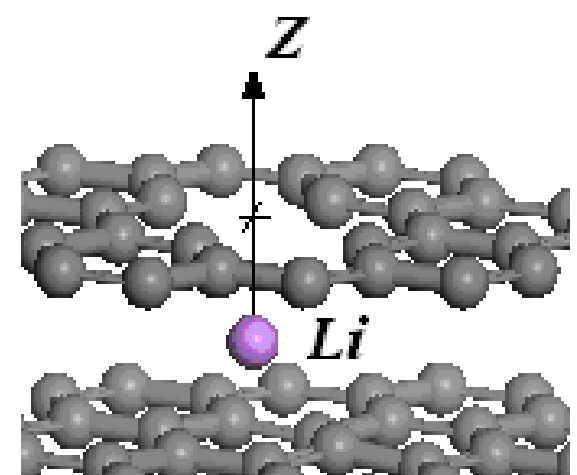

Fig. 12 A possible configuration of two layer graphene with $\mathrm{Li}$ atom encapsulated between them, moving through bi-vacancy.

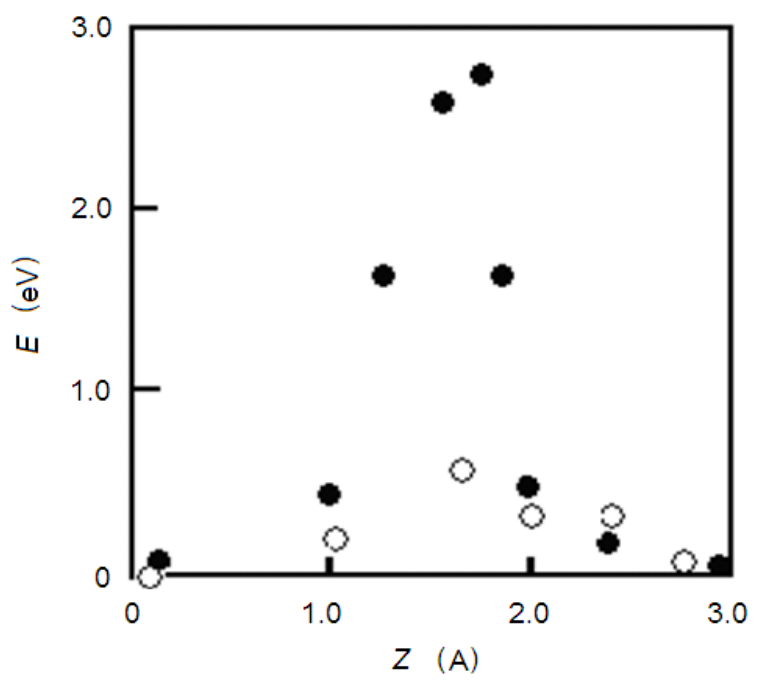

Fig. 13 The energy barrier existing by permeation of $\mathrm{Li}$ through vacancy or bivacancy in grapheme. graphite- and graphene- based elements for storing of Li. It can be thought that it is impossible in principle to improve the state. But some results of computer simulations and calculations of possible configurations of few layer graphene cells contained Li which are given below show that situation becomes much better when graphene sheets of the cell are linked together with a fast bridge-like radiation defect, which makes the cell much more stiffer and improves its dimension stability.

It can be seen that the cell simulated with defect shows no signs of noticeable deformation (Fig. 11). Fig. 11b represents the distribution of electron charge in the area near the bridge-like defect and It can be seen that although there is a possibility of originating the fast bond between $\mathrm{Li}$ and bridge-like defect ( $\mathrm{Li}$ atom on the left side) but mainly two layer graphene cell can be used for nearly full recycling storage of $\mathrm{Li}$.

One more important characteristic of lithium-ion devices is the effective mobility of $\mathrm{Li}$ in the structure of batteries. Therefore, the simulation of possible ways of Li motion is of great interest. In particular, the possibility of direct passing of $\mathrm{Li}$ through single vacancy and bivacancy inside graphene cells was investigated. Fig. 12 presents a scheme of possible way for Li passing through holes in graphene sheet.

Fig. 13 represents the results of DFT calculations, using optimization on energy, which show the existing of a potential barrier on the way of $\mathrm{Li}$ atom passing through graphene sheet with a single vacancy (black marks) or bivacancy (empty marks). The zero point of $\mathrm{Z}$ axis is placed in initial position of $\mathrm{Li}$ atom at the center between graphene sheets.

\section{Conclusions}

Computer simulations and quantum mechanical calculations of graphene- and few-layer grapheme-metal compositions have been performed in order to predict their energetic and structural characteristics. It was revealed that radiation modification of few layer graphene might become a 
key technology to improve physical-mechanical properties of ultra-thin graphite particles and few layer graphene structures as reinforcement elements of composites and as nanocells which serve as possible storing elements for lithium atoms in lithium ion batteries production technologies.

\section{References}

[1] D.H. Lim, A.S. Negreira, J. Wilcox, DFT studies on the interaction of deflective graphene supported $\mathrm{Fe}$ and $\mathrm{Al}$ nanoparticles, J. Phys. Chem. C 115 (18) (2011) 8961-8970.

[2] J.J. Palacios, J.F. Rossier, L. Brey, Vacancy induced magnetism in graphene and graphene ribbons, Phys. Rev. B 77 (2008) 195428-195444.

[3] A.M. Ilyin, Computer Simulation of Radiation Defects in Graphene and Relative Structures, Graphene Simulation,
In Tech, Rijeka, 2011, pp. 39-52.

[4] A.M. Ilyin, E.A. Daineko, G.W. Beall, Computer simulation and study of radiation defects in graphene, Physica E 42 (2009) 67-69.

[5] A.M. Ilyin., G.W. Beall, I.A. Tsyganov, Simulation and study of bridge-like radiation defects in the carbon nanostructures, J. Comp. Theor. Nanosci. 7 (2010) 2004-2007.

[6] A.M Ilyin, G.W. Beall, Computer simulation of graphene-metal composite induced by radiation, in: Nano Tech Conference \& Expo, 2011, pp. 574-576.

[7] K. Zhao, M. Pharr, S. Cai, J.J. Vlassak, Z. Suo, Large plastic deformation in high-capacity lithium ion batteries caused by charge and discharge, J. Am. Ceram. Soc. 94 (2011) 226-235.

[8] A.V. Murugan, T. Muraliganth, A. Manthiram, Rapid, facile microwave-solvothermal synthesis of graphene nanosheets and their polyaniline nanocomposites for energy storage, Chem. Mater. 21 (2009) 5004. 